\title{
Classification of Isolates from the Pseudomonas fluorescens Complex into Phylogenomic Groups Based in Group-Specific Markers
}

\author{
Daniel Garrido-Sanz, Eva Arrebola, Francisco Martínez-Granero, Sonia García-Méndez, \\ Candela Muriel, Esther Blanco-Romero, Marta Martín, Rafael Rivilla and \\ Miguel Redondo-Nieto *
}

Departamento de Biología, Facultad de Ciencias, Universidad Autónoma de Madrid, Madrid, Spain

\section{OPEN ACCESS}

Edited by:

Martha E. Trujillo,

University of Salamanca, Spain

Reviewed by:

Youn-Sig Kwak,

Gyeongsang National University,

South Korea

David Dowling

Institute of Technology Carlow, Ireland

${ }^{*}$ Correspondence:

Miguel Redondo-Nieto miguel.redondo@uam.es

Specialty section:

This article was submitted to Evolutionary and Genomic

Microbiology

a section of the journa

Frontiers in Microbiology

Received: 02 December 2016 Accepted: 27 February 2017

Published: 15 March 2017

Citation:

Garrido-Sanz D, Arrebola E Martínez-Granero F, García-Méndez S, Muriel C, Blanco-Romero E, Martín M, Rivilla $R$ and Redondo-Nieto M (2017) Classification of Isolates from the Pseudomonas fluorescens Complex into Phylogenomic Groups Based in Group-Specific Markers.

Front. Microbiol. 8:413. doi: 10.3389/fmicb.2017.00413
The Pseudomonas fluorescens complex of species includes plant-associated bacteria with potential biotechnological applications in agriculture and environmental protection. Many of these bacteria can promote plant growth by different means, including modification of plant hormonal balance and biocontrol. The P. fluorescens group is currently divided into eight major subgroups in which these properties and many other ecophysiological traits are phylogenetically distributed. Therefore, a rapid phylogroup assignment for a particular isolate could be useful to simplify the screening of putative inoculants. By using comparative genomics on $71 P$. fluorescens genomes, we have identified nine markers which allow classification of any isolate into these eight subgroups, by a presence/absence PCR test. Nine primer pairs were developed for the amplification of these markers. The specificity and sensitivity of these primer pairs were assessed on 28 field isolates, environmental samples from soil and rhizosphere and tested by in silico PCR on 421 genomes. Phylogenomic analysis validated the results: the PCR-based system for classification of $P$. fluorescens isolates has a $98.34 \%$ of accuracy and it could be used as a rapid and simple assay to evaluate the potential of any $P$. fluorescens complex strain.

Keywords: Pseudomonas fluroescens complex, PCR, phylogroups, classification

\section{INTRODUCTION}

The Pseudomonas fluorescens complex of species is one of the most diverse groups within the Pseudomonas genus, comprising more than fifty validly named species and many unclassified isolates. Members of this group have been isolated from diverse habitats, including water (Mirand and Zemelman, 2002), soil (Andersen et al., 2000), plant tissues (Brown et al., 2012), fungi (Rainey et al., 1993), animals (Vela et al., 2006), and humans (Scales et al., 2015). Many P. fluorescens strains that have been isolated from plant-related environments are described as plant growth-promoting rhizobacteria (PGPR) due to their ability to influence plant hormonal balance (Kang et al., 2006) and improve plant fitness by minimizing the effects of phytopathogens (Raaijmakers et al., 2009), for which they are of great biotechnological interest. Pseudomonads are also known for the utilization of diverse organic compounds as energy and carbon sources (Lessie and Phibbs, 1984), making them also suited for bioremediation of polluted environments (Wasi et al., 2013). Despite 
their beneficial role as PGPR, certain species within the $P$. fluorescens complex are pathogens, including Pseudomonas corrugata and Pseudomonas mediterranea, the causal agents of pith necrosis in tomato (Catara, 2007; Trantas et al., 2015) and Pseudomonas tolaasii, which causes brown blotch disease on cultivated mushrooms (Rainey et al., 1993).

An analysis of the $P$. fluorescens complex carried out 25 years ago identified several biotypes by using different taxonomic criteria (Stanier et al., 1966; Palleroni, 1991). More recently multilocus sequence analysis (MLSA) has divided the $P$. fluorescens complex into a varying number of groups depending on the number and identity of the genomes included in the study (Mulet et al., 2010; Gomila et al., 2015; Garrido-Sanz et al., 2016). These MLSA-based analysis have shown good concordance with phylogenomics and comparative genomics (Garrido-Sanz et al., 2016), in which eight phylogroups have been identified: $P$. mandelii, P. jessenii, $P$. koreensis, $P$. corrugata, $P$. fluorescens, $P$. gessardii, P. chlororaphis, and Pseudomonas protegens.

We have previously shown that many ecophysiological traits that are important for biocontrol, plant growth-promotion and bioremediation are distributed phylogenetically among the main groups within the P. fluorescens complex (Garrido-Sanz et al., 2016). For instance, strains within the $P$. corrugata, $P$. chlororaphis, and $P$. protegens groups produce an array of secondary metabolites with antifungal properties, such as 2,4 diacetylphloroglucinol, 2-hexyl, 5-propyl resorcinol, phenazines, and other siderophore-based antibiotics such as pyrrolnitrin and pyoluteorin (Nowak-Thompson et al., 1999; Raaijmakers and Weller, 2001; Mavrodi et al., 2006; Ramette et al., 2011; Loper et al., 2012; Calderón et al., 2013). It has also been reported that insecticidal activity of $P$. fluorescens complex strains also follows a phylogenetic distribution, being present in $P$. protegens and $P$. chlororaphis phylogroups (Flury et al., 2016). On the other hand, strains from $P$. chlororaphis and P. koreensis also carry the biosynthetic gene cluster for indole-3-acetic acid metabolism (Loper et al., 2012; Garrido-Sanz et al., 2016), which enhances plant root system and thus increase the nutrient uptake by the plant (Spaepen et al., 2007). Therefore, routinely phylogroup identification of isolates from the P. fluorescens complex could be a preliminary step toward obtaining strains with potential biotechnological applications.

Scarpellini et al. (2004) first developed a 16S rRNA PCR-based assay to identify $P$. fluorescens isolates and their corresponding biotype. Several genotipes on DAPG-producing fluorescent pseudomonads were also identified by PCR using the BOX1AR primer (BOX-PCR; Weller et al., 2007). Presently, the increase in the number of full sequenced genomes allows the use of phylogenetic and comparative genomic methods, which have provided a more robust insight regarding the delineation of groups within the P. fluorescens complex of species (Mulet et al., 2010; Gomila et al., 2015; Garrido-Sanz et al., 2016). Different PCR-based systems have been developed for the identification of pathogenic $P$. fluorescens complex species, such as $P$. tolaasii (Lee et al., 2002), P. corrugata, and P. mediterranea (Catara et al., 2000; Licciarcdello et al., 2011). Although a rpoD-based PCR procedure also exists for Pseudomonas species identification (Mulet et al., 2009), given that currently the P. fluorescens complex is composed by more of 50 named species and that most of the phylogroups contain several species (Garrido-Sanz et al., 2016) it is necessary to develop a system that allows the identification of these mayor P. fluorescens complex phylogroups, as group adscription can provide insights into the potential biotechnological uses of a particular isolate.

The present study aims to develop a rapid PCR-based system assay for routinely classification of $P$. fluorescens isolates into the eight phylogroups in which it is currently divided (GarridoSanz et al., 2016). For this purpose, we have used comparative genomics to identify specific markers of these groups and develop sets of primers for their amplification. We have tested this system on classified and unclassified Pseudomonas strains along with environmental and rhizosphere samples. Finally, we performed in silico PCR (is PCR) and phylogenomic analysis to theoretically test and validate the system in all the sequenced genomes available.

\section{MATERIALS AND METHODS}

\section{Datasets, Markers Identification, and Primer Design}

Seventy-one genomes and proteomes previously reported of belonging to the $P$. fluorescens complex (Garrido-Sanz et al., 2016) were downloaded from the NCBI FTP server (ftp.ncbi.nih.gov) in February 2015 (Supplementary Table 1). Orthologous groups were identified by comparing all-againstall using BLASTP (Camacho et al., 2009) and processed with OrthoMCL v4 pipeline (Li et al., 2003), using default settings, $50 \%$ alignment coverage cut-off and $1 e-5 e$-value. The results were stored in a relational database for further analysis and filtered with own designed SQL queries and Python scripts to obtain the CDSs from the protein entries that appeared in all the genomes of each group but not in the remaining genomes. These CDSs were additionally filtered by $500 \mathrm{pb}$ minimum length and blasted against all the genomes. The sequences with no hits across genomes outside the groups and high homology within genomes of the group were selected. The selected markers sequences were then retrieved from all the group's genomes and aligned using Clustal Omega (Sievers and Higgins, 2014). Conserved regions were used to design primers. Degenerated bases were introduced to guarantee annealing in all the target genomes. Melting temperature of the primers, absence of dimerization and hairpin formation and lack of secondary priming sites were assessed with OligoAnalyzer 3.1 (https://eu.idtdna.com/ calc/analyzer).

\section{In silico PCR (isPCR) and Phylogenomic Analysis}

Sequences of the nine selected markers plus $100 \mathrm{nts}$ from each end were blasted against nt and wgs NCBI databases on March 2016. Blast hits were processed and reviewed to test primer specificity based on mismatches, specially in $3^{\prime}$ and predicted amplicon length.

The 225 genomes selected from is PCR as being part of any of the 8 P. fluorescens phylogroups (Figure 1) along with 196 


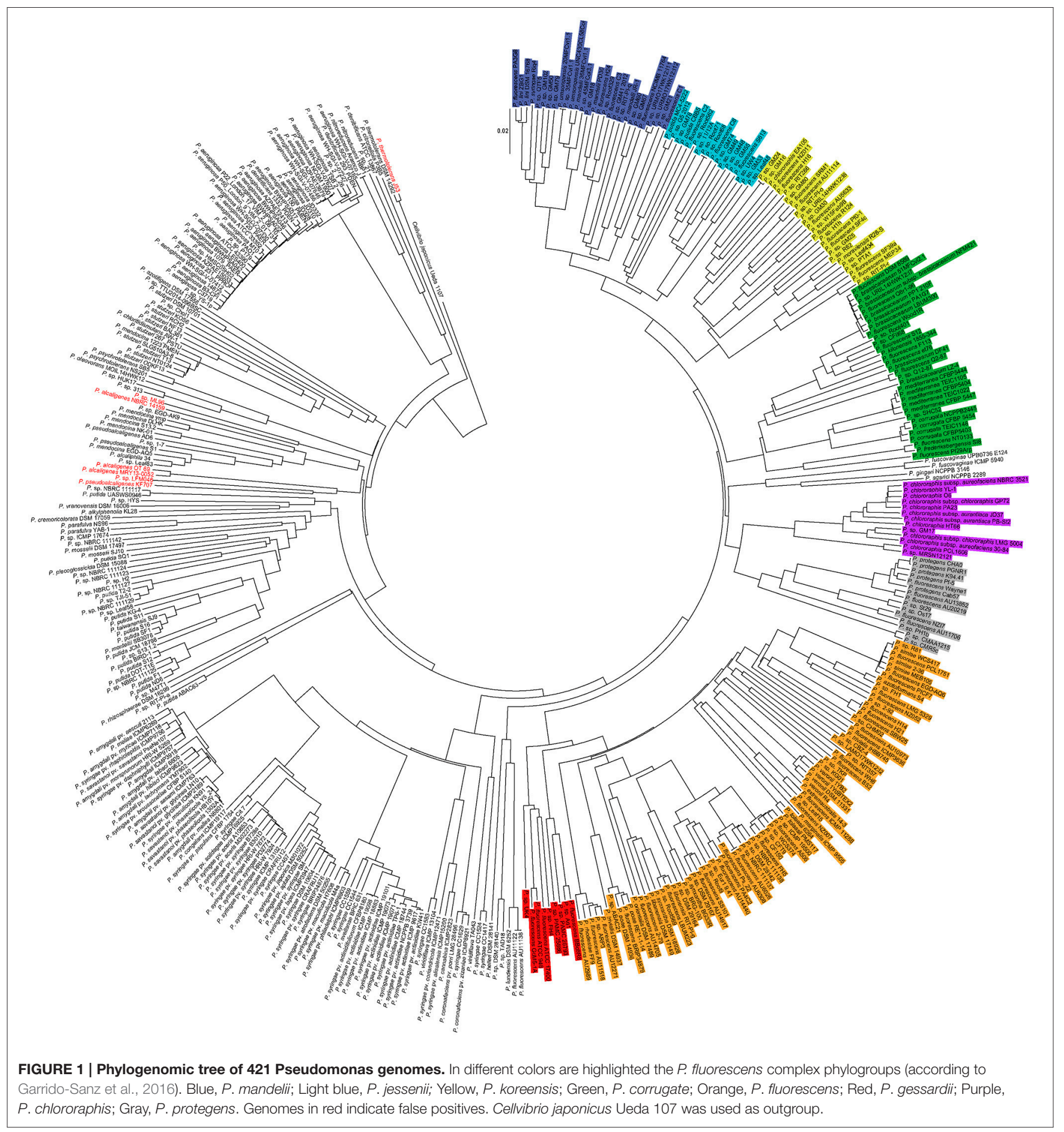

randomly sampled genomes from the remaining Pseudomonas genomes (retrieved from NCBI ftp server on March, 2016; Supplementary Table 1) were compared all-against-all using the Genome-to-genome distance calculator (GGDC) 2.1 web service at http://ggdc.dsmz.de (Meier-Kolthoff et al., 2013). Resulting sets of intergenomic distances (Supplementary File 1) were used to construct a distance matrix with own designed Python scripts.
Phylogenomic trees were built using Neighbor-joining with MEGA v7 software (Kumar et al., 2016).

\section{Pseudomonas Strains Isolation}

Rhizospheric and endophytic pseudomonads were isolated from pepper (C. annuum), tomato (S. lycopersicum), lettuce (L. sativa), and pumpkin (Cucurbita sp.). Non-lignified roots were collected, 
cleaned out from soil remains, introduced in sterile tubes with $25 \mathrm{ml}$ of saline solution $(\mathrm{NaCl} 0.85 \%)$ and vortexed for $5 \mathrm{~min}$ to detach soil residues. For rhizospheric pseudomonads isolation, $100 \mu \mathrm{l}$ of serial dilutions from the saline solution resuspension were plated in SA medium (Scher and Baker, 1982) supplemented with $\mathrm{MgSO}_{4}$, ampicillin $(100 \mu \mathrm{g} / \mathrm{ml})$ and cycloheximide (100 $\mu \mathrm{g} / \mathrm{ml}$ ) and incubated at $28^{\circ} \mathrm{C}$ for $24 \mathrm{~h}$. For endophytic pseudomonads isolation, roots vortexed in saline solution were further surface-sterilized with $10 \%$ sodium hypochlorite for $5 \mathrm{~min}$, then $10 \%$ ethanol for $5 \mathrm{~min}$ and finally rinsed five times with sterile distilled water for $3 \mathrm{~min}$ each. To check complete disinfection, $100 \mu \mathrm{l}$ from the last wash water was plated on LB medium. Disinfected roots were macerated aseptically in saline solution and then filtered. One hundred microliters of the extract were spreaded on SA plates supplemented with $\mathrm{MgSO}_{4}$, ampicillin $(100 \mu \mathrm{g} / \mathrm{ml})$ and cycloheximide $(100 \mu \mathrm{g} / \mathrm{ml})$ and incubated at $28^{\circ} \mathrm{C}$ for $72 \mathrm{~h}$. Fluorescent colonies under UV light from both rhizospheric and endophytic protocols were re-plated and grown at 28 and $37^{\circ} \mathrm{C}$ for $24 \mathrm{~h}$. Those unable to grow at $37^{\circ} \mathrm{C}$ were selected. Additionally, rhizosphere samples 1 and 2 where collected from onion (Allium cepa) and lettuce respectively, and were obtained as above but instead of isolates, the whole plates where swept, followed by total DNA extraction (see below).

Strains 7.3 and 3.2 were isolated by standard enrichment culture techniques, using an inoculum of $1 \mathrm{~g}$ of soil collected in a petrol station located in Tres Cantos (Madrid, Spain). A mineral medium (Brazil et al., 1995), containing biphenyl as the sole carbon source was used for enrichment and purification of the culture as described previously (Chang et al., 2013).

\section{Strains Cultures, DNA Extraction, and PCR Conditions}

All the strains used in this work were grown in LB medium (Luria-Bertrani) at $28^{\circ} \mathrm{C}, 1.5 \%(\mathrm{w} / \mathrm{v})$ purified agar for solid medium. DNA extraction from strains cultures was carried out using the Realpure Genomic DNA Extraction Kit (Durviz, Spain). Metagenomic DNA from $1 \mathrm{~g}$ soil was extracted using the FastDNA ${ }^{\circledR}$ SPIN Kit for Soil (MP Biomedicals, USA).

PCRs were carried out in a total volume of $25 \mu \mathrm{l}$ containing $2.5 \mu \mathrm{l}$ of 10X PCR buffer $\mathrm{MgCl}_{2}$ free, $1 \mu \mathrm{l} \mathrm{MgCl}_{2} 50 \mathrm{mM}, 0.5$ $\mu \mathrm{l}$ dNTP mix $10 \mathrm{mM}(2.5 \mu \mathrm{M}$ each $), 1 \mu \mathrm{l}$ of each primer at 10 $\mu \mathrm{M}, 1 \mu \mathrm{l}$ of Taq DNA polymerase $1 \mathrm{U} / \mu \mathrm{l}$ (Biotools) and $1 \mu \mathrm{l}$ of DNA template 30-50 $\mathrm{ng} / \mu \mathrm{l}$. The cycling conditions consisted in a first denaturation step at $95^{\circ} \mathrm{C}$ for $5 \mathrm{~min}$ followed by 30 cycles of amplification ( $30 \mathrm{~s}$ denaturation at $95^{\circ} \mathrm{C}, 30 \mathrm{~s}$ of primer annealing at $64^{\circ} \mathrm{C}$ for DGPf_0, DGPf_1, DGPf_2, DGPf_3, DGPf_4, and DGPf_7 or $67^{\circ} \mathrm{C}$ for DGPf_5, DGPf_6 and DGPf_8 primer sets, and an elongation step at $72^{\circ} \mathrm{C}$ for $1.5 \mathrm{~min}$ ) followed by a final elongation step at $72^{\circ} \mathrm{C}$ for $7 \mathrm{~min}$. For the metagenomic DNA of environmental samples, the number of cycles was increased to 40 . PCR products were electrophoretically separated in $1.5 \%$ $(\mathrm{w} / \mathrm{v})$ agarose gels and post-dyed with GelRed.

\section{S rDNA Cloning}

16S rDNA from the strains described in Supplementary File 2, was amplified using the universal primers $27 \mathrm{~F}\left(5^{\prime}\right.$ AGAGTTTGATCMTGGCTCAG-3') and 1492R (5'-CTA
CGRRTACCTTGTTACGAC-3'). PCR conditions as described above, using an annealing temperature of $56^{\circ} \mathrm{C}, 25$ cycles, and using $1 \mu \mathrm{l}$ of Ultratools DNA polymerase (Biotools) 1 $\mathrm{U} / \mu 1$. PCR results were cloned into $\mathrm{pGEM}^{\circledR}{ }_{-} \mathrm{T}$ Easy Vector System I (Promega) and transformed into E. coli DH5 $\alpha$. Plasmid DNA was extracted using the kit Wizard ${ }^{\circledR}$ Plus SV Minipreps DNA Purification System (Promega). Inserts were sequenced using the universal primers T7 and SP6. All the 16S rDNA sequences obtained in this study were submitted to the GenBank and are available under the accession numbers specified in Supplementary File 2.

\section{RESULTS AND DISCUSSION}

\section{Nine Markers Are Sufficient to Identify the Eight $P$. fluorescens Phylogroups}

In order to identify markers that allow the classification of a given strain into one of the eight $P$. fluorescens phylogroups, all-againstall genome comparisons were performed on $71 \mathrm{P}$. fluorescens complex genomes (Supplementary Table 1) representing all these groups. The subsequent filtering for highly conserved coding sequences belonging to the core genome of each of the eight phylogroups revealed that the combination of nine markers in a pattern of presence and/or absence are enough for their discrimination (Table 1). Interestingly, the putative function of most of these genes, three of which are transcriptional regulators, does not seem to confer any distinctive group phenotype, but are rather involved in general functions. It is important to notice that adscription of any strain to a given group requires, at least, two positive reactions.

Strains from the $P$. corrugata phylogroup can be identified with the markers DGPf_0 and DGPf_1 (Table 1). While DGPf_0 codifies for a hypothetical protein (PSF113_RS56935) that is also present in $P$. koreensis, $P$. mandelii, and some strains from $P$. jessenii and $P$. protegens phylogroups, DGPf_1 codifies for a type I secretion target (PSF113_RS30625) that was only found in strains belonging to the $P$. corrugata group. Primer pairs for both markers were designed to amplify the regions between nucleotides 139 to 750 for DGPf_0 and from 632 to 1,316 for DGPf_1, resulting in amplicons of 621 and 685 bp respectively (Table 2).

For the P. koreensis phylogroup, aside from DGPf_0, the marker DGPf_2 was identified (Table 1). This second marker encodes for a FAD dependent oxydoreductase (PFL01_RS10805) and primer pair was designed to amplify the region from nucleotides 88 to 1,088 , producing a fragment of $1,001 \mathrm{bp}$ (Table 2). DGPf_2 was also present in all the strains from $P$. jessenii, $P$. protegens and $P$. chlororaphis phylogroups.

For the $P$. jessenii phylogroup, the markers DGPf_2 and DGPf_3 were designed to identify this group strains (Table 1). DGPf_3 encodes for a glutamine synthetase (PputUW4_01890) which primer pair was designed in the 671 to 1,351 region, resulting in a $681 \mathrm{bp}$ amplicon (Table 2). Additionally, marker DGPf_0 was detected in most of the strains from this group (i.e., P. sp. UW4, P. sp. GM33, P. sp. GM55, P. sp. GM49, P. sp. GM74, P. sp. GM78 and P. sp. GM48, and P. sp. G5). In any case, either 
TABLE 1 | P. fluorescens complex specific markers and their distribution within the phylogroups.

Name Gene name (accs. no.) ${ }^{b}$

P. fluorescens complex group ${ }^{a}$

P. corrugata P. koreensis P. jessenii P. mandelii P. gessardii P. fluorescens P. protegens P. chlororaphis

DGPf_0 Hypothetical protein (PSF113_RS556935)

DGPf_1 Type I secretion target (PSF113_RS30625)

DGPf_2 FAD dependent oxidoreductase (PFL01_RS10805)

DGPf_3 Glutamine synthetase (PputUW4_01890)

DGPf_4 KWG repeat-containing protein (PFL_RS20920)

DGPf_5 3-phosphoshikimate 1-carboxyvinyltransferase (PFLA506_RS14455)

DGPf_6 LysR family transcriptional regulator (PFL_RS18605)

DGPf_7 LysR family transcriptional regulator (PFLU_RS11255)

DGPf_8 LuxR family transcriptional regulator (PCL1606_12410)

(n)

$\begin{array}{cccc}+ & + & \pm & + \\ + & - & - & - \\ - & + & + & - \\ - & - & + & + \\ - & - & & -\end{array}$

The presence of the marker in all the strains of a group is indicated with a plus (+) while absence is indicated with a minus (-). Groups in which the marker can be present or absent depending on the strain is indicated with plus/minus ( \pm ).

${ }^{a}$ Groups according to Garrido-Sanz et al. (2016).

${ }^{b}$ Accession number of the most representative gene sequence within each group.

\section{TABLE 2 | Primers designed in this work.}

\begin{tabular}{|c|c|c|c|c|c|}
\hline Marker name & Primer name & Position $^{a}$ & Sequence $\left(5^{\prime}-3^{\prime}\right)$ & $\operatorname{Tm}\left({ }^{\circ} \mathrm{C}\right)$ & Length (bp) \\
\hline DGPf_O & DGPf_OR & & GAAAGTCTTGACCAGCARVAG & & \\
\hline & DGPf_1R & & CCRAGGAAGCCCAGGGAN & & \\
\hline DGPf_2 & DGPf_2F & $88 . .1088$ & GTRGTSTTCATCGGBGGHGG & 64 & 1001 \\
\hline & DGPf_3R & & GCAGTTCCCAGTCGGTKATBCGYCGGTCG & & \\
\hline \multirow[t]{2}{*}{ DGPf_4 } & DGPf_4F & $32 . .1103$ & CGCTGATCCTCTCGTTGTCTGC & 64 & 1072 \\
\hline & DGPf_4R & & ACGCCCTTGTCCACATCG & & \\
\hline \multirow[t]{2}{*}{ DGPf_5 } & DGPf_5F & $3 . .1117$ & CGGCGTGGGTGTCGATCRR & 67 & 1115 \\
\hline & DGPf_5R & & GAGTTCGCAGAAAACCGTGACCG & & \\
\hline DGPf_7 & DGPf_7R & & GCTGAARTCTGGVAGCAGGGC & & \\
\hline \multirow[t]{2}{*}{ DGPf_8 } & DGPf_8F & $127 . .787$ & CCCACCGACAGCCAGCAACG & 67 & 661 \\
\hline & DGPf_8R & & CGGTCTTGTCGCTGATGCCG & & \\
\hline
\end{tabular}

Degenerated bases are indicated in bold.

a Target marker position for the primers according to genes described in Table 1.

if a certain strain results positive for the amplification of markers DGPf_2 and DGPf_3, or for both of them and DGPf_0, there is no other phylogroup that can be misidentified with these markers combinations (Table 1).
The $P$. mandellii phylogroup can be identified with the combination of DGPf_0 and DGPf_3 markers as described above, and the lack of the DGPf_2 one (Table 1). 
For the $P$. protegens phylogroup, aside from DGPf_2, the marker DGPf_4 was identified (Table 1). The DGPf_4 marker encodes for a KWG repeat-containing protein (PFL_RS20920) and primer set was developed to amplify the region between nucleotides 32 and 1,103, which amplifies a fragment of 1,072 bp (Table 2). Additionally, markers DGPf_0 and DGPf_6 were also present on some of the strains from this group (Table 1).

For the $P$. gessardii phylogroup, markers DGPf_5 and DGPf_6 were identified (Table 1). The marker DGPF_5 encodes for a 3-phosphoshikimate 1-carboxyvinyltransferase (PFLA506_RS14455) whose primer pair was developed to amplify the region between nucleotides 3 and $1,117 \mathrm{pb}$, resulting in an amplicon of 1,115 bp (Table 2). This marker is also present in all the strains from $P$. fluorescens phylogroup. On the other hand, DGPf_6 encodes for a LysR family transcriptional regulator (PFL_RS18605) and aside from all the $P$. gessardii strains it is also present in some $P$. protegens phylogroup strains. Primer set was designed to amplify a $680 \mathrm{bp}$ fragment from nucleotide 28 to nucleotide $707 \mathrm{pb}$ of this gene (Table 2).

For the $P$. fluorescens phylogroup, aside from DGPf_5, the specific DGPf_7 marker was identified (Table 1). This marker encodes for another LysR family transcriptional regulator (PFLU_RS11255) and primer set was designed in the 11 to $755 \mathrm{pb}$ region, resulting in a 745 bp fragment amplification (Table 2).

Finally, for the $P$. chlororaphis phylogroup, the combination of DGPf_2 and the specific DGPf_8 markers are able to identify strains belonging to this group. DGPf_8 encodes for a LuxR family transcriptional regulator (PCL1606_12410) and primer set was designed to amplify the region between 127 to $787 \mathrm{pb}$ (Table 2).

As expected, given the genetic heterogeneity of the $P$. fluorescens complex genomes, we did not find any highly conserved specific marker for the P. koreensis, P. jessenii, P. mandelii, and P. gessardii phylogroups. However, the combination of two different markers allows their correct affiliation. On the other hand, $P$. corrugata, $P$. fluorescens, $P$. protegens, and $P$. chlororaphis groups do have markers that are exclusive to them. Therefore, the combination of the presence and/or absence of these nine markers allows the classification of a given strain into one of these groups with at least two different markers present in each of them (Table 1).

Primer sequences were aligned against all the genomes from their group. When mismatches where detected, degenerated bases were introduced to ensure optimal primer hybridization. Primer sequences and their marker target regions for PCR amplification are listed in Table 2. After in silico PCR (see below), primer sequences were checked again and degenerated bases were introduced to maximize theoretical hybridization in the 147 additional P. fluorescens complex genomes identified.

\section{PCR Test of Model $P$. fluorescens Strains}

Primer sets and annealing temperatures were empirically tested in gradient temperature PCRs in nine model $P$. fluorescens complex strains (P. fluorescens F113, P. fluorescens Q2-87, P. fluorescens Q8r1-96 (renamed P. brassicacearum Q8r1-96), $P$. brassicacearum NFM421, $P$. chlororaphis PCL 1319, $P$. chlororaphis PCL1606, P. protegens Pf-5, P. fluorescens Pf0-1, and
$P$. fluorescens SBW25) belonging to five different phylogroups (P. corrugata, $P$. chlororaphis, $P$. protegens, $P$. koreensis, and $P$. fluorescens respectively). The optimal annealing temperatures are specified in Table 2. Additionally, three other Pseudomonas model strains, outside the $P$. fluorescens complex were tested as negative controls ( $P$. putida KT2440, $P$. syringae pv. tomato DC3000, and P. aeruginosa PAO1) along with Escherichia coli $\mathrm{DH} 5 \alpha$. All the PCRs for the P. fluorescens complex strains resulted in positive amplification for the markers of the groups they belong to (Table 3), with amplicon sizes congruent with the theoretically expected. For the negative controls, no amplification was observed with the exception of the DGPf_2 primer set in $P$. putida KT2440 (Table 3) that does not allow the classification of the strain as belonging to any $P$. fluorescens phylogroup.

\section{Blind PCR Test of $P$. fluorescens Isolates and Environmental Samples}

Seventeen putative $P$. fluorescens isolates from rhizosphere and endosphere of pepper (Capsicum annuum), tomato (Solanum lycopersicum), lettuce (Lactuca sativa), and pumpkin (Cucurbita sp.) along with two other soil isolates (Supplementary Table 2) were tested for their phylogroup affiliation. PCR results unequivocally assigned each isolate to a single phylogroup. Six (31.6\%) of the isolates belonged to the P. koreensis phylogroup, another six (31.6\%) for the P. jessenii phylogroup, five $(26.3 \%)$ isolates were placed in the $P$. fluorescens phylogroup, and two (10.5\%) were classified in $P$. corrugata phylogroup (Table 4). No isolate from $P$. chlororaphis, $P$. protegens, $P$. mandelii, or $P$. gessardii groups were found. $16 \mathrm{~S}$ rDNA sequence of 10 of these strains was obtained (EMC3, EMC5, RMT7, 3.2, RMT1, RMT2, EMC7, RMT4, RMP9, and 7.3) and showed high sequence identity with strains from the phylogroups they were assigned to by the PCR system (Supplementary File 2). Additionally, wholegenome sequence of four of these isolates (EMC3, 3.2, RMP9, and 7.3) and subsequent phylogenomic analysis validated their correct group affiliation (data not shown).

An environmental soil sample was also tested. Here, four primer sets gave positive results; DGPf_0, DGPf_3, DGPf_5, and DGPf_7 (Table 4), suggesting the presence of DNA from P. mandelii and P. fluorescens phylogroups. Two additional rhizosphere samples (1 and 2) where tested, resulting in amplification for primer sets DGPf_0, DGPf_1, DGPf_2, DGPf_3, DGPf_5, and DGPf_7 for sample 1 and DGPf_0, DGPf_2, DPGf_3, DGPf_5, DGPf_7, and DGPf_8 for sample 2 (Table 4). These primer combination suggest the presence of DNA from P. corrugata, P. koreensis, P. jessenii, P. mandelii, and $P$. fluorescens in sample 1 and $P$. koreensis, $P$. jessenii, $P$. mandelii, $P$. fluorescens, and $P$. chlororaphis in sample 2, although other sources cannot be dismissed. On the other hand, the absence of certain markers in these samples strongly suggests lack of certain phylogroups. For instance, negative amplification of markers DGPf_1, DGPf_2, DGPf_4, DGPf_6, and DGPf_8 in the soil sample makes the presence of $P$. corrugata, $P$. koreensis, $P$. jessenii, $P$. gessardii, $P$. protegens, and $P$. chlororaphis phylogroups unlikely. However, in rhizosphere samples, where most of strains from the $P$. fluorescens complex phylogroups can be found, the 
TABLE 3 | P. fluorescens complex strains and other Pseudomonas tested for their $P$. fluorescens complex phylogroup affiliation.

Strain tested

\begin{tabular}{|c|c|c|c|c|c|c|c|c|c|}
\hline & & & & Markers & & & & & \\
\hline DGPf_0 & DGPf_1 & DGPf_2 & DGPf_3 & DGPf_4 & DGPf_5 & DGPf_6 & DGPf_7 & DGPf_8 & $\begin{array}{l}\text { P. fluorescens } \\
\text { phylogroup identified }\end{array}$ \\
\hline
\end{tabular}

P. fluorescens $\mathrm{F} 113$

P. fluorescens Q2-87

P. fluorescens Q8r1-96

P. brassicacearum NFM421

P. chlororaphis PCL 1319

P. chlororaphis PCL1606

P. protegens Pf-5

P. fluorescens PfO-1

P. fluorescens SBW25

P. putida KT2440

P. syringae pv. tomato DC3000

$P$. aeruginosa $\mathrm{PAO} 1$

E. coli $\mathrm{DH} 5 \alpha$

+
+
+
+
-
-
-
+
-
-
-
-
-

PCR positive result is indicated with a plus (+) while a negative result is indicated with a minus (-).

TABLE 4 | Pseudomonas isolates and environmental samples affiliation to the different $P$. fluorescens complex phylogroups.

\begin{tabular}{|c|c|c|c|c|c|c|c|c|c|c|}
\hline Isolate/sample tested & \multicolumn{9}{|c|}{ Markers } & $\begin{array}{l}\text { P. fluorescens phylogroup } \\
\text { identified }\end{array}$ \\
\hline EMC5 $^{\mathrm{a}}$ & + & - & + & - & - & - & - & - & - & P. koreensis \\
\hline $\mathrm{RMT}^{\mathrm{a}}$ & + & - & + & - & - & - & - & - & - & P. koreensis \\
\hline $3.2^{\mathrm{ab}}$ & + & - & + & - & - & - & - & - & - & P. koreensis \\
\hline EMC11 & + & - & + & + & - & - & - & - & - & P. jessenii \\
\hline RMT1 $1^{\mathrm{a}}$ & + & - & + & + & - & - & - & - & - & P. jessenii \\
\hline $\mathrm{RMT}^{\mathrm{a}}$ & + & - & + & + & - & - & - & - & - & P. jessenii \\
\hline RMC8 & + & - & + & + & - & - & - & - & - & P. jessenii \\
\hline EMT2 & - & - & - & - & - & + & - & + & - & P. fluorescens \\
\hline EMT8 & - & - & - & - & - & + & - & + & - & P. fluorescens \\
\hline $\mathrm{RMT}_{4}^{\mathrm{a}}$ & - & - & - & - & - & + & - & + & - & P. fluorescens \\
\hline RMT12 & - & - & - & - & - & + & - & + & - & P. fluorescens \\
\hline RMPga,b & + & + & - & - & - & - & - & - & - & P. corrugata \\
\hline $7.3^{a, b}$ & + & + & - & - & - & - & - & - & - & P. corrugata \\
\hline Soil sample & + & - & - & + & - & + & - & + & - & P. mandelii and $P$. fluorescens \\
\hline Rhizosphere sample 1 & + & + & + & + & - & + & - & + & - & $\begin{array}{l}\text { P. corrugata, P. koreensis, } \\
\text { P. jessenii, P. mandelii, and } \\
\text { P. fluorescens }\end{array}$ \\
\hline Rhizosphere sample 2 & + & - & + & + & - & + & - & + & + & $\begin{array}{l}\text { P. koreensis, P. jessenii, } \\
\text { P. mandelii, P. fluorescens, } \\
\text { and P. chlororaphis }\end{array}$ \\
\hline
\end{tabular}

${ }^{a}$ Group adscription confirmed by $16 S$ rDNA sequence.

${ }^{b}$ Group adscription confirmed by whole-genome sequence. 
number of primer sets resulting in negative amplification are reduced. This finding makes the system not only suited for testing isolates, but also for testing environmental samples in order to assess the diversity of $P$. fluorescens complex phylogroups that might be present.

\section{In silico PCR and Phylogenomics of Pseudomonas Genomes}

To test the extent of the PCR-based system to reliably characterize new isolates, is PCR was assessed by blasting the nine markers against the whole NCBI nt and wgs databases on March 2016, which included more than 65,000 prokariotic genomes, and checking for the marker combination presence, primer mismatches (specially in $3^{\prime}$ ) and theoretical amplicon length in all genomes. is PCR identified 225 genomes which gave a positive identification pattern (see in detail in Supplementary File 3). This dataset also included the 71 genomes used for generating the markers set. Thirty-two of these genomes (14.22\%) belonged to the P. corrugata phylogroup, $28(12.44 \%)$ to the P. koreensis phylogroup, $23(10.22 \%)$ to the $P$. jessenii phylogroup, 28 (12.44\%) to the P. mandelii phylogroup, 9 (4\%) to the P. gessardii phylogroup, 77 (34.22\%) to the P. fluorescens phylogroup, 15 (6.67\%) to the $P$. protegens phylogroup and $13(5.78 \%)$ to the $P$. chlororaphis phylogroup.

These 225 genomes were further analyzed by phylogenomics in order to corroborate their classification. Another 196 genomes randomly sampled from the remaining 2,310 Pseudomonas genomes available at the NCBI ftp server (ftp.ncbi.nih.gov, March 2016) were also included as negative control. As shown in Figure 1, from the 225 genomes identified as belonging to the P. fluorescens complex by is PCR, 218 (96.9\%) showed total correlation between the PCR data and phylogenomic assignment, indicating that they were correctly placed within each group by the PCR method. The remaining seven genomes $(3.1 \%)$, were false positives and they all belonged to the $P$. aeruginosa lineage (Garrido-Sanz et al., 2016). Two of these genomes (P. sp. ML96, and P. pseudoalcaligenes KF707) were wrongly identified by $i s \mathrm{PCR}$ as belonging to the $P$. koreensis phylogroup, while the other four ( $P$. alcaligenes NBCRC 14159, $P$. sp. LFMO46, $P$. thermotolerans J53, $P$. alcaligenes OT 69 and $P$. alcaligenes MRY13-0052) were wrongly identified as belonging to the $P$. jessenii phylogroup (Supplementary File 3). On the other hand, from the 196 randomly sampled genomes, none of them were shown to be false negatives. In consequence, the isPCR has shown that the system has a $98.34 \%$ of accuracy, as from the 421 genomes tested, just seven were misidentified.

Interestingly, the phylogenomic analysis has also shown the presence of two more phylogroups within the same branch that leads to the $P$. fluorescens complex (Figure 1). The most distal one includes $P$. lundensis DSM 6252, the novel species $P$. helleri (von Neubeck et al., 2016) and other isolates. P. lundensis has been previously reported to belong to the $P$. fragi phylogroup within the P. fluorescens complex (Mulet et al., 2010; Gomila et al., 2015; Garrido-Sanz et al., 2016). However, this phylogroup has only been identified based on MLSA, as no sequenced genome was available at the time of these analysis. For the same reason, this group was not included for the markers identification. The other group, identified as $P$. asplenii group, is shown to be within the branch that leads into the $P$. chlororaphis and $P$. protegens phylogroups. However, none of the previous studies of the Pseudomonas genus place it inside of the $P$. fluorescens complex (Mulet et al., 2010; Gomila et al., 2015; Garrido-Sanz et al., 2016). These discrepancies with previous studies could be due to the larger number of genomes included here. The three species within the $P$. asplenii phylogroup ( $P$. asplenii, $P$. fuscovaginae, and $P$. agarici) are known pathogens of fern, rice and mushrooms respectively (Ark and Tompkins, 1946; Batoko et al., 1994; Gill and Cole, 2000), therefore their adscription to the P. fluorescens complex should be further analyzed. In any case, none of the genomes from these groups resulted in positive amplification for any of the markers. More worrying is the finding that several strains have been wrongly classified at the species level. This is the case of $P$. syringae Riq4, placed now within the $P$. mandelii phylogroup, P. putida strains MC4-5222 and CBB5, which really belong to the $P$. jessenii phylogroup, $P$. chlororaphis EA105, in the $P$. koreensis phylogroup and P. frederiksbergensis SI8 in the P. corrugata phylogroup (Figure 1). All these strains clearly do not belong to the species they have been assigned to, highlighting that more efforts should be done to avoid inaccurate and misleading species naming and the need for databases curing.

\section{CONCLUSIONS}

The results presented here provide an accurate, easy to perform and cheap method for the initial profiling of strains belonging to the $P$. fluorescens complex of species and isolated from plant material or soil. The method allows the placement of each isolate unequivocally in one of the eight phylogroups that have been previously described by MLSA and digital DNA-DNA hybridization. It also allows the assessment of the presence/absence of specific phylogroups in complex samples (metagenomic DNA from soil or plant samples). Considering the phylogenetic distribution of biotechnology relevant traits, the method could be included as one of the first steps in protocols that require the screening of a large number of pseudomonads isolates in the search for inoculants for agriculture or environmental technologies.

\section{AUTHOR CONTRIBUTIONS}

Performed experiments and Bioinformatic analysis: DGS, MR. Strains Isolation and Characterization: DGS, EA, FM, SG, CM, EBR, RR. Manuscript Drafting: DGS, MM, RR, MR. Experimental Design and Supervision: MM, RR, MR.

\section{ACKNOWLEDGMENTS}

Research was funded by grant BIO2015-64480R from MINECO/FEDER EU. DGS was granted by FPU fellowship program (FPU14/03965) from Ministerio de Educación, Cultura 
y Deporte, Spain. EBR is the recipient of a fellowship from Fundación Tatiana Pérez de Guzmán el Bueno, Spain.

\section{SUPPLEMENTARY MATERIAL}

The Supplementary Material for this article can be found online at: http://journal.frontiersin.org/article/10.3389/fmicb. 2017.00413/full\#supplementary-material

Supplementary Table 1 | List of genomes used in this study.

\section{REFERENCES}

Andersen, S. M., Johnsen, K., Sørensen, J., Nielsen, P., and Jacobsen, C. S. (2000). Pseudomonas frederiksbergensis sp. nov., isolated from soil at a coal gasification site. Int. J. Syst. Evol. Microbiol. 50(Pt 6), 1957-1964. doi: 10.1099/00207713-50-6-1957

Ark, P. A., and Tompkins, C. M. (1946). Bacterial leaf blight of bird's-nest fern. Phytopathology 36, 758-761.

Batoko, H., Kinet, J. M., Maraite, H., and Bouharmont, J. (1994). Susceptibility to brown sheath rot of rice correlated to sensitivity to bacterial toxin. Rice Genet. Newsl. 11, 102-103.

Brazil, G. M., Kenefick, L., Callanan, M., Haro, A., de Lorenzo, V., Dowling, D. N., et al. (1995). Construction of a rhizosphere pseudomonad with potential to degrade polychlorinated biphenyls and detection of bph gene expression in the rhizosphere. Appl. Environ. Microbiol. 61, 1946-1952.

Brown, S. D., Utturkar, S. M., Klingeman, D. M., Johnson, C. M., Martin, S. L., Land, M. L., et al. (2012). Twenty-one genome sequences from Pseudomonas species and 19 genome sequences from diverse bacteria isolated from the rhizosphere and endosphere of Populus deltoides. J. Bacteriol. 194, 5991-5993. doi: 10.1128/JB.01243-12

Calderón, C. E., Pérez-García, A., de Vicente, A., and Cazorla, F. M. (2013). The dar genes of Pseudomonas chlororaphis PCL1606 are crucial for biocontrol activity via production of the antifungal compound 2-hexyl, 5-propyl resorcinol. Mol. Plant Microbe Interact. 26, 554-565. doi: 10.1094/MPMI-01-13-0012-R

Camacho, C., Coulouris, G., Avagyan, V., Ma, N., Papadopoulos, J., Bealer, K., et al. (2009). BLAST+: architecture and applications. BMC Bioinformatics 10:421. doi: 10.1186/1471-2105-10-421

Catara, V. (2007). Pseudomonas corrugata: plant pathogen and/or biological resource? Mol. Plant Pathol. 8, 233-244. doi: 10.1111/j.1364-3703.2007.00391.x

Catara, V., Arnold, D., Cirvilleri, G., and Vivian, A. (2000). Specific oligonucleotide primers for the rapid identification and detection of the agent of Tomato Pith Necrosis, Pseudomonas corrugata, by PCR amplification: evidence for two distinct genomic groups. Eur. J. Plant Pathol. 106, 753-762. doi: 10.1023/A:1026507423848

Chang, Y. C., Takada, K., Choi, D., Toyama, T., Sawada, K., and Kikuchi, S. (2013). Isolation of biphenyl and polychlorinated biphenyl-degrading bacteria and their degradation pathway. Appl. Biochem. Biotechnol. 170, 381-398. doi: 10.1007/s12010-013-0191-5

Flury, P., Aellen, N., Ruffner, B., Péchy-Tarr, M., Fataar, S., Metla, Z., et al. (2016). Insect pathogenicity in plant-beneficial pseudomonads: phylogenetic distribution and comparative genomics. ISME J. 10, 2527-2542. doi: 10.1038/ismej.2016.5

Garrido-Sanz, D., Meier-Kolthoff, J. P., Goker, M., Martin, M., Rivilla, R., and Redondo-Nieto, M. (2016). genomic and genetic diversity within the Pseudomonas fluorescens complex. PLoS ONE 11:e0150183. doi: 10.1371/journal.pone.0150183

Gill, W., and Cole, T. (2000). Aspects of the pathology and etiology of 'drippy gill' disease of the cultivated mushroom Agaricus bisporus. Can. J. Microbiol. 46, 246-258. doi: 10.1139/w99-133

Gomila, M., Peña, A., Mulet, M., Lalucat, J., and García-Valdés, E. (2015). Phylogenomics and systematics in Pseudomonas. Front. Microbiol. 6:214. doi: 10.3389/fmicb.2015.00214

Kang, B. R., Yang, K. Y., Cho, B. H., Han, T. H., Kim, I. S., Lee, M. C., et al. (2006). Production of indole-3-acetic acid in the plant-beneficial strain Pseudomonas
Supplementary Table 2 | Pseudomonas isolates used for blind test.

Supplementary File 1 | GGDC intergenomic distances of 421 Pseudomonas genomes.

Supplementary File 2 | Closest-related strain and phylogroup adscription according to $16 \mathrm{~S}$ sequence identity.

Supplementary File 3 | In silico PCR results.

chlororaphis $\mathrm{O} 6$ is negatively regulated by the global sensor kinase GacS. Curr. Microbiol. 52, 473-476. doi: 10.1007/s00284-005-0427-x

Kumar, S., Stecher, G., and Tamura, K. (2016). MEGA7: molecular Evolutionary genetics analysis version 7.0 for bigger datasets. Mol. Biol. Evol. 33, 1870-1874. doi: $10.1093 / \mathrm{molbev} / \mathrm{msw} 054$

Lee, H. I., Jeong, K. S., and Cha, J. S. (2002). PCR assays for specific and sensitive detection of Pseudomonas tolaasii, the cause of brown blotch disease of mushrooms. Lett. Appl. Microbiol. 35, 276-280. doi: 10.1046/j.1472-765X.2002.01178.x

Lessie, T. G., and Phibbs, P. V. Jr. (1984). Alternative pathways of carbohydrate utilization in pseudomonads. Annu. Rev. Microbiol. 38, 359-388. doi: 10.1146/annurev.mi.38.100184.002043

Li, L., Stoeckert, C. J. Jr., and Roos, D. S. (2003). OrthoMCL: identification of ortholog groups for eukaryotic genomes. Genome Res. 13, 2178-2189. doi: 10.1101/gr.1224503

Licciarcdello, G., Bella, P., and Catara, V. (2011). Quantitative detection of Pseudomonas corrugata and Pseudomonas mediterranea in tomato plants by duplex real-time PCR. J. Plant Pathol. 93, 595-602. doi: 10.4454/jpp.v93i3.3642

Loper, J. E., Hassan, K. A., Mavrodi, D. V., Davis, E. W. II., Lim, C. K., Shaffer, B. T., et al. (2012). Comparative genomics of plant-associated Pseudomonas spp.: insights into diversity and inheritance of traits involved in multitrophic interactions. PLoS Genet. 8:e1002784. doi: 10.1371/journal.pgen.1002784

Mavrodi, D. V., Blankenfeldt, W., and Thomashow, L. S. (2006). Phenazine compounds in fluorescent Pseudomonas spp. biosynthesis and regulation. Annu. Rev. Phytopathol. 44, 417-445. doi: 10.1146/annurev.phyto.44.013106.145710

Meier-Kolthoff, J. P., Auch, A. F., Klenk, H. P., and Goker, M. (2013). Genome sequence-based species delimitation with confidence intervals and improved distance functions. BMC Bioinformatics 14:60. doi: 10.1186/1471-2105-14-60

Mirand, C. D., and Zemelman, R. (2002). Antimicrobial multiresistance in bacteria isolated from freshwater Chilean salmon farms. Sci. Total Environ. 293, 207-218. doi: 10.1016/S0048-9697(02)00022-0

Mulet, M., Bennasar, A., Lalucat, J., and García-Valdés, E. (2009). An rpoDbased PCR procedure for the identification of Pseudomonas species and for their detection in environmental samples. Mol. Cell. Probes 23, 140-147. doi: 10.1016/j.mcp.2009.02.001

Mulet, M., Lalucat, J., and Garcia-Valdes, E. (2010). DNA sequence-based analysis of the Pseudomonas species. Environ. Microbiol. 12, 1513-1530. doi: 10.1111/j.1462-2920.2010.02181.x

Nowak-Thompson, B., Chaney, N., Wing, J. S., Gould, S. J., and Loper, J. E. (1999). Characterization of the pyoluteorin biosynthetic gene cluster of Pseudomonas fluorescens Pf-5. J. Bacteriol. 181, 2166-2174.

Palleroni, N. J. (1991). "Present situation of the taxonomy of aerobic Pseudomonas," in Pseudomonas. Molecular Biology and Biotechnology, eds E. Galli, S. Silver, and B. Witholt (Washington, DC: American Society for Microbiology), 105-115.

Raaijmakers, J. M., Paulitz, T. C., Steinberg, C., Alabouvette, C., and MoënneLoccoz, Y. (2009). The rhizosphere: a playground and battlefield for soilborne pathogens and beneficial microorganisms. Plant Soil 321:20. doi: 10.1007/s11104-008-9568-6

Raaijmakers, J. M., and Weller, D. M. (2001). Exploiting genotypic diversity of 2,4-diacetylphloroglucinol-producing Pseudomonas spp.: characterization of superior root-colonizing P. fluorescens strain Q8r1-96. Appl. Environ. Microbiol. 67, 2545-2554. doi: 10.1128/AEM.67.6.2545-2554.2001 
Rainey, P. B., Brodey, C. L., and Johnstone, K. (1993). Identification of a gene cluster encoding three high-molecular-weight proteins, which is required for synthesis of tolaasin by the mushroom pathogen Pseudomonas tolaasii. Mol. Microbiol. 8, 643-652. doi: 10.1111/j.1365-2958.1993.tb01608.x

Ramette, A., Frapolli, M., Fischer-Le Saux, M., Gruffaz, C., Meyer, J. M., Défago, G., et al. (2011). Pseudomonas protegens sp. nov., widespread plant-protecting bacteria producing the biocontrol compounds 2,4diacetylphloroglucinol and pyoluteorin. Syst. Appl. Microbiol. 34, 180-188. doi: 10.1016/j.syapm.2010.10.005

Scales, B. S., Erb-Downward, J. R., LiPuma, J. J., and Huffnagle, G. B. (2015). Draft genome sequences of five Pseudomonas fluorescens subclade, I., and II Strains, isolated from human respiratory samples. Genome Announc. 3:e00837-15. doi: 10.1128/genomeA.00837-15

Scarpellini, M., Franzetti, L., and Galli, A. (2004). Development of PCR assay to identify Pseudomonas fluorescens and its biotype. FEMS Microbiol. Lett. 236, 257-260. doi: 10.1111/j.1574-6968.2004.tb09655.x

Scher, F. M., and Baker, R. (1982). Effects of Pseudomonas putida and a synthetic iron chelator on induction of soil supressiveness to Fusarium wilt pathogens. Phytopathology 72, 1567-1573. doi: 10.1094/Phyto-72-1567

Sievers, F., and Higgins, D. G. (2014). Clustal Omega, accurate alignment of very large numbers of sequences. Methods Mol. Biol. 1079, 105-116. doi: 10.1007/978-1-62703-646-7_6

Spaepen, S., Vanderleyden, J., and Remans, R. (2007). Indole-3-acetic acid in microbial and microorganism-plant signaling. FEMS Microbiol. Rev. 31, 425-448. doi: 10.1111/j.1574-6976.2007.00072.x

Stanier, R. Y., Palleroni, N. J., and Doudoroff, M. (1966). The aerobic pseudomonads: a taxonomic study. J. Gen. Microbiol. 43, 159-271. doi: 10.1099/00221287-43-2-159

Trantas, E. A., Licciardello, G., Almeida, N. F., Witek, K., Strano, C. P., Duxbury, Z., et al. (2015). Comparative genomic analysis of multiple strains of two unusual plant pathogens: Pseudomonas corrugata and Pseudomonas mediterranea. Front. Microbiol. 6:811. doi: 10.3389/fmicb.2015.00811

Vela, A. I., Gutiérrez, M. C., Falsen, E., Rollan, E., Simarro, I., Garcia, P., et al. (2006). Pseudomonas simiae sp. nov., isolated from clinical specimens from monkeys (Callithrix geoffroyi). Int. J. Syst. Evol. Microbiol. 56(Pt 11), 2671-2676. doi: 10.1099/ijs.0.64378-0

von Neubeck, M., Huptas, C., Gluck, C., Krewinkel, M., Stoeckel, M., Stressler, T., et al. (2016). Pseudomonas helleri sp. nov. and Pseudomonas weihenstephanensis sp. nov., isolated from raw cow's milk. Int. J. Syst. Evol. Microbiol. 66, 1163-1173. doi: 10.1099/ijsem.0.000852

Wasi, S., Tabrez, S., and Ahmad, M. (2013). Use of Pseudomonas spp. for the bioremediation of environmental pollutants: a review. Environ. Monit. Assess. 185, 8147-8155. doi: 10.1007/s10661-013-3163-x

Weller, D. M., Landa, B. B., Mavrodi, O. V., Schroeder, K. L., De La Fuente, L., Blouin Bankhead, S., et al. (2007). Role of 2,4-diacetylphloroglucinol-producing fluorescent Pseudomonas spp. in the defense of plant roots. Plant Biol. 9, 4-20. doi: $10.1055 /$ s-2006-924473

Conflict of Interest Statement: The authors declare that the research was conducted in the absence of any commercial or financial relationships that could be construed as a potential conflict of interest.

Copyright (C) 2017 Garrido-Sanz, Arrebola, Martínez-Granero, García-Méndez, Muriel, Blanco-Romero, Martín, Rivilla and Redondo-Nieto. This is an open-access article distributed under the terms of the Creative Commons Attribution License (CC BY). The use, distribution or reproduction in other forums is permitted, provided the original author(s) or licensor are credited and that the original publication in this journal is cited, in accordance with accepted academic practice. No use, distribution or reproduction is permitted which does not comply with these terms. 